J.P. KOTTMANN O.J.F. MARTIN

\section{Influence of the cross section and the permittivity on the plasmon-resonance spectrum of silver nanowires}

Electromagnetic Fields and Microwave Electronics Laboratory, Swiss Federal Institute of Technology, ETZ G96, ETH-Zentrum, 8092 Zurich, Switzerland

\begin{abstract}
Received: 15 August 2001
Published online: 10 October 2001 • (C) Springer-Verlag 2001

ABSTRACT We investigate the plasmon resonances for silver nanowires with a non-regular cross section. To study the relationship between the cross section and the spectrum of the plasmon resonances, we consider cross sections evolving from a rectangular shape to a triangular one. In particular, we study the influence of the sharpness of a corner on the near-field enhancement at the vicinity of a particle and discuss its implications for surface-enhanced Raman scattering. We also investigate the influence of the absorption on the plasmon-resonance spectrum and on the near-field enhancement.
\end{abstract}

PACS 73.20.Mf; 78.67.-n; 87.64.Je

1

Introduction

The excitation, at optical frequencies, of surfaceplasmon resonances in metallic nanoparticles is probably one of the most spectacular optical effects in metals.

These plasmon resonances lead to extremely strong fields at the vicinity of a nanoparticle. Such fields play for example a key role in surface-enhanced Raman scattering (SERS), wherein the Raman signal of a molecule deposited on the nanoparticle is enhanced by several orders of magnitude $[1,2]$. This enhancement can be large enough that the Raman spectrum of a single molecule can be detected [3-5].

The large scattering cross section (SCS) associated with the plasmon resonance also produces a strong signal in the far field, so that nanoscopic particles can be easily detected. This was recently used to label specific targets on DNA, using functionalized plasmon-resonant particles [6].

As the plasmon resonances occur for specific wavelengths, they also give the particle its color. This strong, frequency-specific signal was already used in the middle ages, as craftsmen incorporated metallic particles into glass paste to give stained glass windows the remarkable colors we can still enjoy today. This however remained an art close to alchemy; especially, the relation between the metallic particle shape and its color, i.e. its spectrum of plasmon resonances, was not known.

Fax: +41-1/632-1647, E-mail: martin@ifh.ee.ethz.ch
In the 19th century, Faraday noted that there must be a relationship between the particle size and its spectrum of plasmon resonances [7]. The investigation of this problem remained however extremely limited, as only very simple shapes, like a sphere or an ellipsoid in 3D $[8,9]$, or a nanowire with a cylindrical or elliptical section in 2D [8], can be solved using Mie theory or related semi-analytical techniques. The resonances of a cube have been computed by Fuchs in the electrostatic limit [10], while particles larger than $100 \mathrm{~nm}$ have been investigated numerically by different groups [11-13]. However, these calculations become very difficult when the particle size is smaller than $100 \mathrm{~nm}$, as extremely rapid variations of the field occur on very short distances [14].

Since particles in the $20-50 \mathrm{~nm}$ range appear to produce the strongest SERS enhancement and the sharpest resonances [15], we recently developed a very stable finite-element technique to compute the optical response of plasmon-resonant nanoparticles in this range [16].

Using this technique to study the resonance spectra of silver nanowires, we showed that nanowires with a non-regular cross section had a complex spectrum, with many different resonances [17]. In particular, triangular wires seem to produce the most complex spectrum. This was confirmed in [18], where we systematically investigated nanowires with different convex polygonal sections.

In these previous works, we noticed the role played by corners in the plasmon resonances of non-regular-shape particles. In particular, extremely strong fields occur at the vicinity of the corners, when the resonances occur. However, this effect should not be mistaken for a lightning-rod effect, which can also produce some field enhancement at the particle corner, although much weaker than that induced by the plasmon resonance [19].

The objective of the present paper is twofold. First, we shall investigate in detail the influence of the particle corners on the spectrum of plasmon resonances and the associated near-field enhancement. Second, we will study the impact of the permittivity function that enters our model on the width and strength of the plasmon resonances.

The paper is organized as follows: in Sect. 2 we briefly recall our computation model. The numerical results are presented in Sect. 3 and a summary is given in Sect. 4 . 
2

\section{Model}

We consider a homogeneous nanowire oriented along the $z$ axis, with an arbitrary cross section and a complex permittivity $\varepsilon(\omega)=\varepsilon^{\prime}(\omega)+\mathrm{i} \varepsilon^{\prime \prime}(\omega)$, where $\omega$ is the frequency. The wire is placed in an infinite homogeneous background $\varepsilon_{\mathrm{B}}=1$. This system is invariant in the $z$ direction and we can limit its study to a 2D $x y$ cross section (Fig. 1). The wire is illuminated with an incident plane wave propagating in the $x y$ plane, with the electric field $\boldsymbol{E}^{0}(\boldsymbol{r})$ polarized in the same plane (so-called transverse electric (TE) polarization, Fig. 1). Throughout the paper, we consider an incident field propagating along the (11) direction (Fig. 1).

To describe the response of the wire, we must compute the total scattered field $\boldsymbol{E}(\boldsymbol{r})$, which is a solution of the integral equation [20]

$$
\begin{aligned}
\boldsymbol{E}(\boldsymbol{r} ; \omega)= & \boldsymbol{E}^{0}(\boldsymbol{r} ; \omega) \\
& +\int_{V} \mathrm{~d} \boldsymbol{r}^{\prime} \boldsymbol{G}^{\mathrm{B}}\left(\boldsymbol{r}, \boldsymbol{r}^{\prime} ; \omega\right) k_{0}^{2}\left(\varepsilon\left(\boldsymbol{r}^{\prime} ; \omega\right)-\varepsilon_{\mathrm{B}}\right) \boldsymbol{E}\left(\boldsymbol{r}^{\prime} ; \omega\right),
\end{aligned}
$$

where $k_{0}^{2}=\omega^{2} / c^{2}$ is the vacuum wave number, $\boldsymbol{G}^{\mathrm{B}}\left(\boldsymbol{r}, \boldsymbol{r}^{\prime} ; \omega\right)$ the Green's tensor associated with the infinite homogeneous background and $V$ the wire section [20].

To solve (1) numerically, we discretize the wire section using finite elements defined on triangular meshes (Fig. 1). Depending on the complexity of the problem, we use between 3000 and 5000 meshes, with a higher refinement towards the particle corners (Fig. 1). Note that we round off the particle corner by $0.25 \mathrm{~nm}$, to provide a more realistic model and avoid numerical instabilities related to sharp corners. This procedure does not affect the accuracy of the results [18].

We refer the reader to [16], where our computational technique is described and assessed in detail. Let us however briefly mention one attractive feature of this approach: the integral formulation in (1) allows us to limit the discretization of the system to the wire section, even when the field

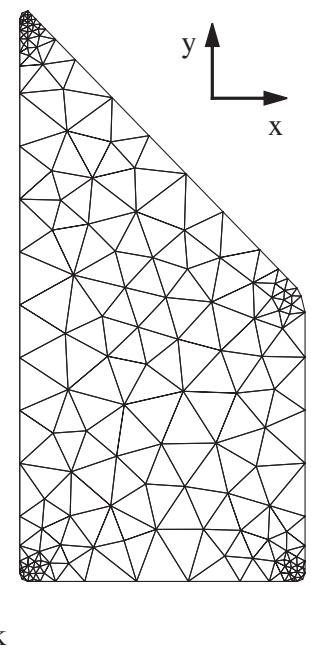

FIGURE 1 A nanowire with a non-regular cross section is illuminated with an incident field $\boldsymbol{E}^{0}$ propagating along the $\boldsymbol{k}$ direction. To compute the scattered field using (1), the cross section of the wire is discretized using 3000-5000 triangular meshes

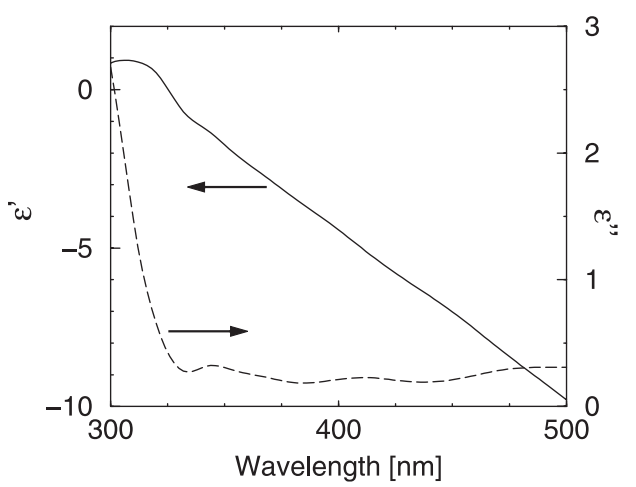

FIGURE 2 Dispersion relation for silver used in our calculations. The real $\varepsilon^{\prime}$ and the imaginary $\varepsilon^{\prime \prime}$ parts of the permittivity are interpolated from the experimental data of Johnson and Christy [23]

outside the wire is computed (note that the term $\varepsilon(\boldsymbol{r} ; \omega)-\varepsilon_{\mathrm{B}}$ vanishes when $\boldsymbol{r} \notin V$ ). This can be extended to more complex backgrounds, when the particle is for example deposited on a surface or embedded in a stratified medium: also in those cases, only the particle must be discretized [21, 22].

One of the most important ingredients that enter (1) is the permittivity $\varepsilon(\omega)$. For our calculations we use the experimental data of Johnson and Christy [23], as shown in Fig. 2. Since this parameter governs the response of the particle to an external field, we shall discuss in Sect. 3.2 its influence on the plasmon resonances.

\section{3}

\section{Results}

\section{1}

\section{Cross-sectional shape}

In order to understand the special role played by corners in the plasmon-resonance spectrum, we investigate particles of similar size but varying form. Figure 3 shows the SCS for nanowires with sections that evolve from a rectangular shape into a triangular one. The triangular section is such that it has the same area as a cylinder with a $20 \mathrm{~nm}$ diameter; the base is approximately $17 \mathrm{~nm}$ and the height $34 \mathrm{~nm}$. The rectangle has the same dimensions and the intermediate particles have a short side with length $0.75,0.5$, respectively 0.25 that of the triangle height (Fig. 3).

In Fig. 3 we observe that the main resonance is red-shifted from $373 \mathrm{~nm}$ (rectangle) to $470 \mathrm{~nm}$ (triangle), as the particle changes. Since the overall particle size shrinks, one would rather expect a blue shift of the main resonance [15].

Additional resonances appear as the shape of the particle evolves, leading to at least four well-separated resonances for the triangular particle (Fig. 3). Note that the resonance around $329 \mathrm{~nm}$ visible for the rectangular particle is also present for the triangular one, although its magnitude is much smaller. This peculiar resonance, which takes place where $\varepsilon(\omega) \simeq 0$ (Fig. 2) and produces a near-field enhancement in the direction longitudinal to the illumination propagation (see Fig. 4(e) in [17]), must be related to the bulk plasmon [17]. The strength of the SCS associated with this resonance appears to be proportional to the forward path in the particle, in the illumination direction. This path is longer for the rectangular particle than for the triangular one, which explains the weaker SCS observed in the latter case. On the other hand, when the triangular particle is illuminated along its hypotenuse [illumination 


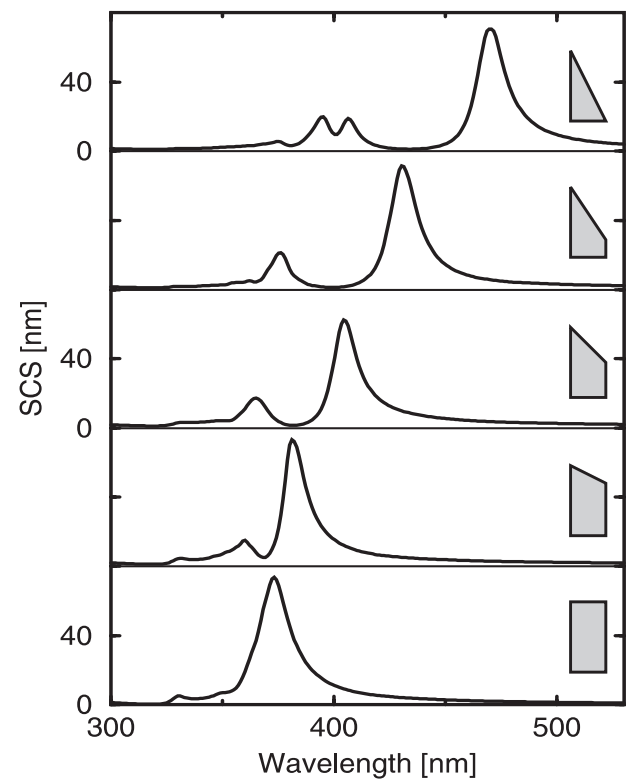

FIGURE 3 SCS for nanowires with a cross section evolving from a rectangular shape (bottom) to a triangular shape (top). The corresponding shape is shown in the inset; see the text for the dimensions. Illumination direction as indicated in Fig. 1; the same vertical scale is used for the five curves

direction $(2 \overline{1})]$, the resonance at $329 \mathrm{~nm}$ is stronger, as the longitudinal path can extend along the hypotenuse.

The full-width at half maximum (FWHM) for the main resonances in Fig. 3 is in the order of $15 \mathrm{~nm}$ and, surprisingly, does not vary much with the particle shape. It is however difficult to correlate the FWHM with the particle geometry only, as the main resonance occurs at a different wavelength, i.e. for a different permittivity. For example the particle with the least-cut corner (Fig. 3, second from the bottom) has the narrowest resonance $(\mathrm{FWHM}=14 \mathrm{~nm})$, but this resonance takes place at the wavelength where the absorption is minimum $\left(\varepsilon^{\prime \prime}=0.18\right)$, which narrows the resonance.

Whereas the SCS gives the overall response of the particle, the near-field enhancement at short distances from the particle surface plays a key role in several practical applications. It is for example responsible for non-radiative energy transfer between neighboring particles [24-26]. The near field also produces the SERS effect when molecules are adsorbed on the particle $[1,2,27]$.

At the plasmon resonance, except for the longitudinal resonance at $329 \mathrm{~nm}$ discussed previously, it is the particle corners transverse to the illumination direction that light up with a strong near field (see Fig. 4a in [17]). For the particles shown in Fig. 3, it is the top-left corner that produces the strongest field at the resonance (except for the $392 \mathrm{~nm}$ resonance of the triangular particle, where the field enhancement is the largest at the lower-right corner).

In Fig. 4 we show the field-amplitude enhancement, with respect to the illumination amplitude, as a function of the distance from the top-left corner. For each particle, we observe that the field rapidly decays as one moves away from the particle surface. This spatial dependence is very important for the SERS effect, as the enhancement effectively exhibited by a molecule depends on the field magnitude at the location of its active Raman site. This site can be at a certain
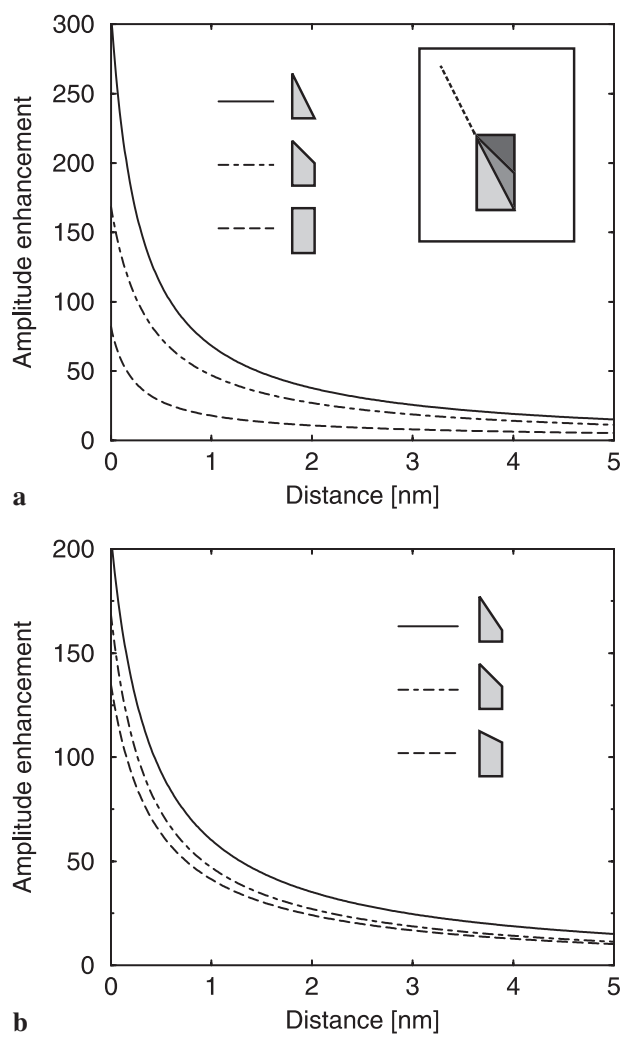

FIGURE 4 Field-amplitude enhancement as a function of the distance from the corner (see inset), for different particles, at the corresponding main resonance wavelength $\lambda$ (see Fig. 3). a Triangle $(\lambda=470 \mathrm{~nm})$, intermediate polygon $(\lambda=403 \mathrm{~nm})$ and rectangle particles $(\lambda=373 \mathrm{~nm}) ; \mathbf{b}$ three different intermediate particles $(\lambda=432 \mathrm{~nm}, \lambda=403 \mathrm{~nm}$ and $\lambda=382 \mathrm{~nm})$

distance from the particle surface. Further, minute shifts of the molecule position within the strong field gradients can lead to variations of the SERS signal. This type of molecular blinking has actually been observed in single-molecule SERS experiments [4].

Although the magnitude of the SCS is similar for the different particles (Fig. 3), we observe in Fig. 4 that the near-field enhancement is much stronger for the triangular particle than for the rectangular one. At a $0.5 \mathrm{~nm}$ distance from the corner, the field amplitude is 112, 66 and 35 times that of the illumination for the triangular, intermediate polygonal and rectangular particles, respectively (Fig. 4a). As the Raman enhancement is proportional to the fourth power of the amplitude enhancement [2], these values correspond to a Raman enhancement in the order of $1.6 \times 10^{8}, 1.8 \times 10^{7}$ and $1.7 \times 10^{6}$, respectively. At larger distances from the surface, the amplitude remains stronger for the triangular particle. A similar behavior is observed for the intermediate polygonal sections reported in Fig. 4b. Overall, the triangular wire gives the strongest enhancement. For comparison, note that the corresponding field-amplitude enhancement for a cylindrical nanowire is only $5.8\left(1.1 \times 10^{3}\right.$ Raman $)$.

These strong fields at the vicinity of the particle corner can be related to oscillating polarization charges of the same sign accumulating at the corner [28]. The sharper the corner, the more confined the charges and the stronger the resulting field. A more complex charge distribution occurs for the next resonance, as plus and minus charges accumulate in the corner. This 

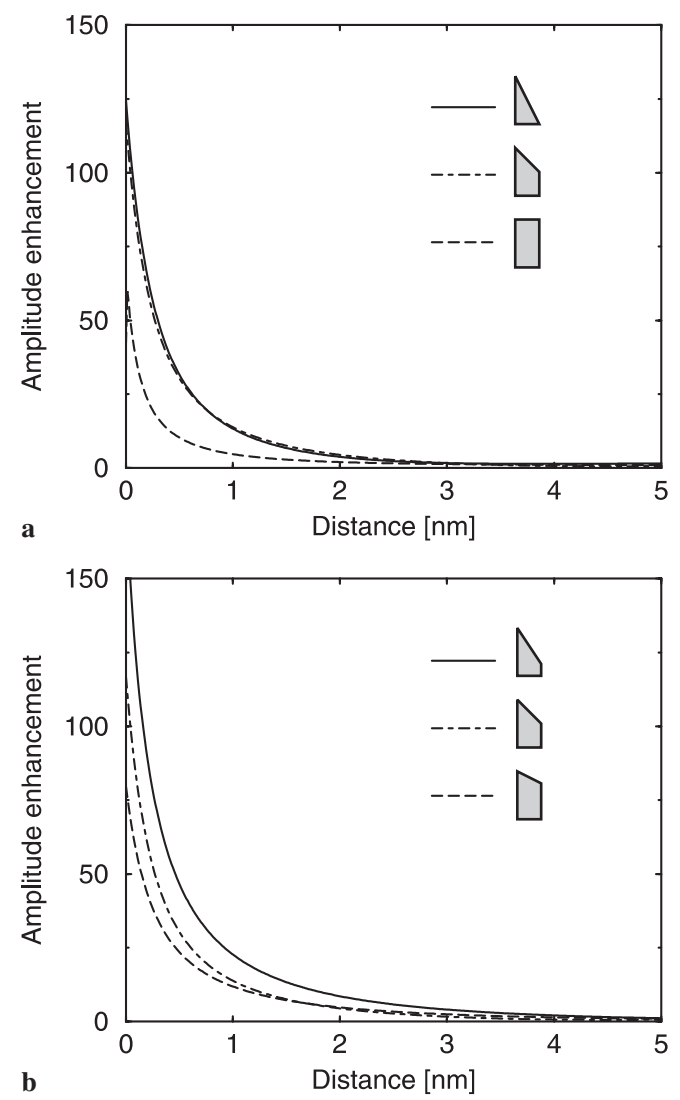

FIGURE 5 Field-amplitude enhancement as a function of the distance from the corner (see inset in Fig. 4), for different particles, at the corresponding second resonance wavelength $\lambda$ (see Fig. 3). a Triangle $(\lambda=404 \mathrm{~nm})$, intermediate polygon $(\lambda=366 \mathrm{~nm})$ and rectangle particles $(\lambda=354 \mathrm{~nm})$; b three different intermediate particles $(\lambda=376 \mathrm{~nm}, \lambda=366 \mathrm{~nm}$ and $\lambda=360 \mathrm{~nm})$

dipolar-like distribution produces a faster-decaying field outside the particle, as illustrated in Fig. 5. Surprisingly, in that case, the strongest near field is not obtained for the triangular particle, but for the nearest polygonal particle (Fig. 5b). This effect is a complex combination between the particle shape, which dictates the resonance condition and how polarization charges can be confined at a corner, and the permittivity at the corresponding resonance. For this second resonance, the particle exhibiting the strongest near field is also that where the resonance occurs at a frequency where $\varepsilon^{\prime \prime}$ is minimum (see Fig. 3, second from the top, and Fig. 2).

\section{2}

\section{Wire permittivity}

The previous results emphasize how complex the study of plasmon resonances can be. In particular, the comparison between different particle shapes is quite delicate, as the corresponding resonances occur at specific frequencies, with different permittivities. In particular, $\varepsilon^{\prime \prime}-$ which accounts for the absorption - strongly influences the resonance width and the near-field enhancement. To clarify this point, we shall concentrate in this section on the triangular particle and study the influence of the absorption on the SCS and near-field enhancement.

The definition of the permittivity for a nanoscopic particle is not trivial. For particles smaller than $5 \mathrm{~nm}$, the classical description breaks down and jellium or quantumchemistry models must be used $[29,30]$. For the particles in the $10-30 \mathrm{~nm}$ range considered here, a local permittivity can still be used to describe the metal, but the bulk value must be modified, as the particle size becomes comparable to the bulk electron mean free path (the average path of a conduction electron between two scattering processes). In that case, electron scattering at the particle surface becomes a dominant effect. It reduces the electron mean free path, which in turn leads to an increase of the imaginary part of the permittivity. This has been evidenced experimentally for spherical particles by Kreibig et al. [31-33]. They showed that for particle sizes below $40 \mathrm{~nm}$ the resonance width becomes broader, and in particular they found that near the plasma-resonance frequency the imaginary part can be well described by the relation

$\varepsilon^{\prime \prime}=0.23+2.64 / a$,

where $a$ is the particle size in nanometers $[8,31]$.

More recently, the electron dynamics in silver and copper particles in the $5-10 \mathrm{~nm}$ range was investigated using femtosecond laser pulses [34]. It was found that for a $6.5 \mathrm{~nm}$ particle size the resonance width is between three and four times larger than expected from the bulk permittivity, thereby indicating a larger absorption. The resonance was also slightly blue-shifted, indicating that the real part of the permittivity can also be affected for such a small particle size [34]. Similar results have been obtained for silver particles in the $3-13 \mathrm{~nm}$ range by another group [35]. For spherical particles in the $20 \mathrm{~nm}$ range investigated here, only the imaginary part of the permittivity seems to be affected by the particle size [ 8 , 33].

No experimental data are available for the modification of the dispersion relation in non-spherical particles of a given shape. One can however expect that corners produce additional electron scattering, thereby locally increasing the absorption. Following (2), and to gain insights into the influence of the absorption on the particle response, we shall investigate three different absorptions.

In Fig. 6, we show the SCS for the triangular wire, using three different values for the imaginary part $\varepsilon^{\prime \prime}$ of the permittivity. The original values of Johnson and Christy, as measured for the bulk, produce four well-resolved resonances. The main resonance is quite narrow $(\mathrm{FWHM}=16 \mathrm{~nm})$. This resonance broadens when the absorption increases: it is FWHM $=28 \mathrm{~nm}$ for $\varepsilon^{\prime}+\mathrm{i} 2 \varepsilon^{\prime \prime}$ and FWHM $=52 \mathrm{~nm}$ for $\varepsilon^{\prime}+\mathrm{i} 4 \varepsilon^{\prime \prime}$ (Fig. 6).

Further, the absorption has a strong influence on the higher-order resonances, which progressively merge into one broad resonance, as the imaginary part of the permittivity increases. Note in Fig. 6 that the resonances are not shifted, since only the imaginary part of the permittivity changes (the plasmon-resonance condition being solely determined by the real part of the permittivity).

In Fig. 7, we study the influence of the damping factor on the near-field enhancement. This effect is quite dramatic, as the field amplitude for the main resonance at $0.5 \mathrm{~nm}$ from the surface drops from $112\left(\varepsilon^{\prime}+\mathrm{i} \varepsilon^{\prime \prime}\right)$ to $65\left(\varepsilon^{\prime}+\mathrm{i} 2 \varepsilon^{\prime \prime}\right)$ and $36\left(\varepsilon^{\prime}+\mathrm{i} 4 \varepsilon^{\prime \prime}\right)$. The effect of the particle permittivity remains visible at larger distances from the surface (Fig. 7a). For the 


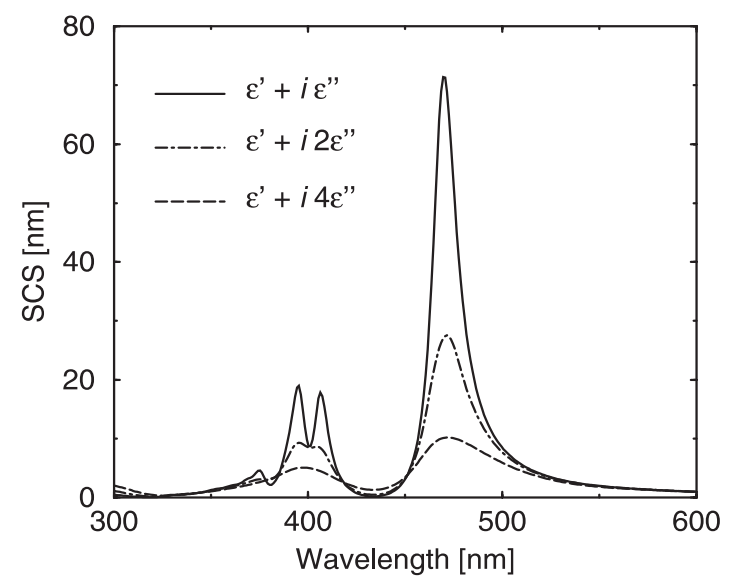

FIGURE 6 SCS for the right-angled triangle, using different permittivities with increasing imaginary parts (absorption factor)
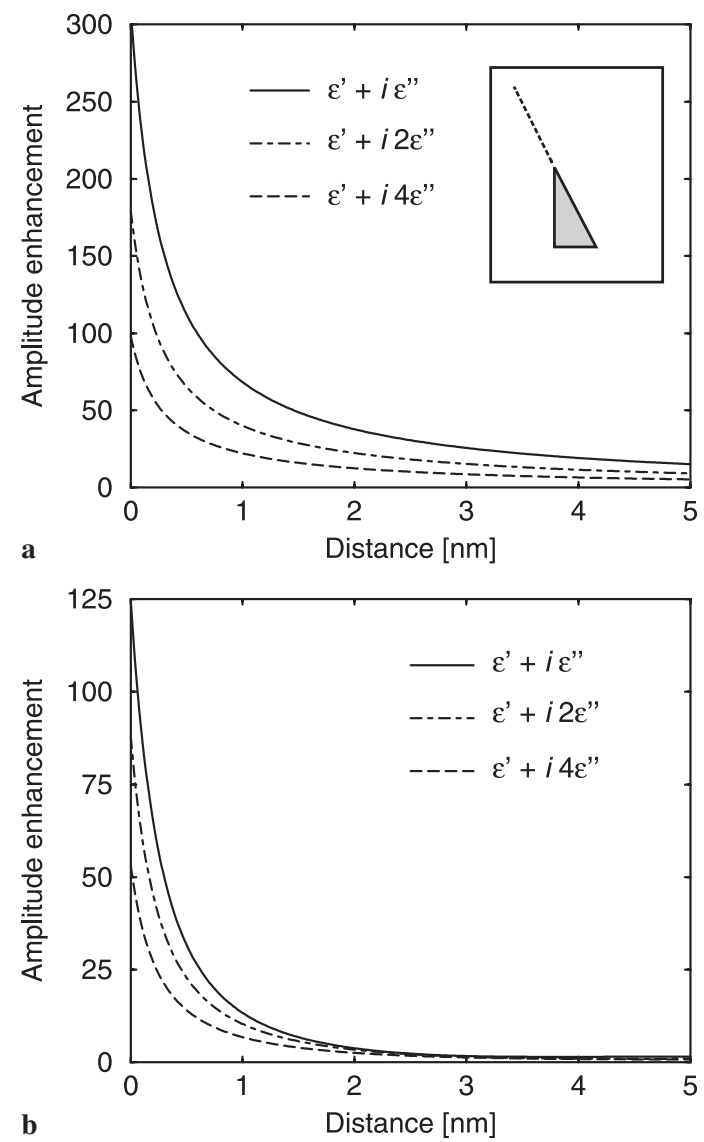

FIGURE 7 Field-amplitude enhancement as a function of the distance from the corner (see inset) for the right-angled triangle, using different permittivities with increasing imaginary parts (absorption factor). Two resonances are investigated: a main resonance $(\lambda=470 \mathrm{~nm})$ and $\mathbf{b}$ next-order resonance $(\lambda=404 \mathrm{~nm})$

next resonance, a similar behavior is observed as a function of the damping, although the field rapidly vanishes at short distances from the particle (Fig. 7b).

\section{$4 \quad$ Conclusion}

We have studied the plasmon-resonance spectrum for silver nanowires with a non-regular cross section. Our results indicate that the overall particle shape determines the different plasmon resonances that can be excited. Progressively breaking the particle symmetry does not continuously increase the number of resonances, but additional resonances appear for specific particle shapes. In particular, triangular particles lead to the largest number of resonances.

Although the magnitude of the scattering cross section can be comparable for the main resonances of similar particles, large differences in the near-field enhancement are observed. Again the triangular particle produces the strongest enhancement. This localized field enhancement corresponds to a SERS enhancement in excess of $10^{8}$. These effects are however very sensitive to the absorption in the metal, and an increase of the imaginary part of the permittivity leads to a much weaker near-field enhancement, as well as to broader resonances.

Our results also illustrate the complexity of plasmonresonance engineering to determine the optimal particle geometry to produce a resonance at a specific wavelength. As the particle shape changes, the frequency where a given resonance occurs shifts. The corresponding material permittivity being different, the resonance width and the near-field enhancement can be strongly modified.

ACKNOWLEDGEMENTS It is a pleasure to acknowledge extremely stimulating discussions with D.R. Smith and S. Schultz from University of California in San Diego. This work was supported by the Swiss National Science Foundation.

\section{REFERENCES}

1 H. Metiu: Prog. Surf. Sci. 17, 153 (1984)

2 M. Moskovits: Rev. Mod. Phys. 57, 783 (1985)

3 K. Kneipp, Y. Wang, H. Kneipp, L.T. Perelman, I. Itzkan, R.R. Dasari, M.S. Feld: Phys. Rev. Lett. 78, 1667 (1997)

4 S. Nie, S.R. Emory: Science 275, 1102 (1997)

5 H. Xu, J. Aizpurua, M. Käll, P. Apell: Phys. Rev. E 62, 1 (2000)

6 S. Schultz, D.R. Smith, J.J. Mock, D.A. Schultz: Proc. Natl. Acad. Sci. USA 97, 996 (2000)

7 M. Kerker: Appl. Opt. 30, 4699 (1991)

8 C.F. Bohren, D.R. Huffman: Absorption and Scattering of Light by Small Particles (Wiley, New York 1983)

9 S. Asano: Appl. Opt. 18, 712 (1979)

10 R. Fuchs: Phys. Rev. B 11, 1732 (1975)

11 W.-H. Yang, G.C. Schatz, R.P. van Duyne: J. Chem. Phys. 103, 869 (1995)

12 T.R. Jensen, G.C. Schatz, R.P. van Duyne: J. Phys. Chem. B 103, 2394 (1999)

13 N. Félidj, J. Aubard, G. Lévi: J. Chem. Phys. 111, 1195 (1999)

14 T.R. Jensen, L. Kelly, A. Lazarides, G.C. Schatz: J. Cluster Sci. 10, 295 (1999)

15 J.P. Kottmann, O.J.F. Martin, D.R. Smith, S. Schultz: Opt. Express 6, 213 (2000)

16 J.P. Kottmann, O.J.F. Martin: IEEE Trans. Antennas Propag. 48, 1719 (2000)

17 J.P. Kottmann, O.J.F. Martin, D.R. Smith, S. Schultz: Chem. Phys. Lett. 341, 1 (2001)

18 J.P. Kottmann, O.J.F. Martin, D.R. Smith, S. Schultz: Phys. Rev. B, in press (2001)

19 J. van Bladel: Singular Electromagnetic Fields and Sources (Clarendon, Oxford 1991)

20 O.J.F. Martin, N.B. Piller: Phys. Rev. E 58, 3909 (1998)

21 M. Paulus, P. Gay-Balmaz, O.J.F. Martin: Phys. Rev. E 62, 5797 (2000)

22 M. Paulus, O.J.F. Martin: J. Opt. Soc. Am. A 18, 854 (2001)

23 P.B. Johnson, R.W. Christy: Phys. Rev. B 6, 4370 (1972) 
24 J.R. Krenn, A. Dereux, J.-C. Weeber, E. Bourillot, Y. Lacroute, J.-P. Goudonnet, G. Schider, W. Gotschy, A. Leitner, F.R. Aussenegg, C. Girard: Phys. Rev. Lett. 82, 2590 (1999)

25 J.-C. Weeber, A. Dereux, C. Girard, J.R. Krenn, J.-P. Goudonnet: Phys. Rev. B 60, 9061 (1999)

26 J.R. Krenn, J.-C. Weeber, E. Bourillot, A. Dereux, J.-P. Goudonnet, G. Schider, A. Leitner, F.R. Aussenegg, C. Girard: Phys. Rev. B 60, 5029 (1999)

27 F.J. García-Vidal, J.B. Pendry: Phys. Rev. Lett. 77, 1163 (1996)

28 J.P. Kottmann, O.J.F. Martin, D.R. Smith, S. Schultz: New J. Phys. 2 , $27.1(2000)$
29 M. Brack: Rev. Mod. Phys. 65, 677 (1993)

30 V. Bonacic-Koutecky, P. Fantucci, J. Koutecky: Chem. Rev. 91, 1035 (1991)

31 U. Kreibig, C. von Fragstein: J. Phys. F 4, 999 (1974)

32 L. Genzel, T.P. Martin, U. Kreibig: Z. Phys. B 21, 339 (1975)

33 U. Kreibig, M. Vollmer: Optical Properties of Metal Clusters (Springer Ser. Mater. Sci. 25) (Springer, Berlin 1995)

34 J.-Y. Bigot, V. Halté, J.C. Merle, A. Daunois: Chem. Phys. 251, 181 (2000)

35 N.D. Fatti, F. Vallée, C. Flytzanis, Y. Hamanaka, A. Nakamura: Chem. Phys. 251, 215 (2000) 